\title{
Vibrational and electronic entropy of $\beta$-cerium and $\gamma$-cerium measured by inelastic neutron scattering
}

\author{
Michael E. Manley, ${ }^{1}$ Robert J. McQueeney, ${ }^{1}$ Brent Fultz, ${ }^{2}$ Raymond Osborn, ${ }^{3}$ George H. Kwei, ${ }^{1}$ and Peter D. Bogdanoff ${ }^{2}$ \\ ${ }^{1}$ Los Alamos National Laboratory, Los Alamos, New Mexico 87545 \\ ${ }^{2}$ Division of Engineering and Applied Science, California Institute of Technology, Pasadena, California 91125 \\ ${ }^{3}$ Argonne National Laboratory, Argonne, Illinois 60439 \\ (Received 26 October 2001; published 1 April 2002)
}

\begin{abstract}
Time-of-flight (TOF) inelastic neutron-scattering spectra were measured on $\beta$-cerium (double hcp) and $\gamma$-cerium (fcc) near the phase-transition temperature. Phonon densities of states (DOS) and crystal-field levels were extracted from the TOF spectra. A softening of the phonon DOS occurs in the transition from $\beta$ - to $\gamma$-cerium, accounting for an increase in vibrational entropy of $\Delta S_{\mathrm{vib}}^{\gamma-\beta}=(0.09 \pm 0.05) k_{B} /$ atom. The entropy calculated from the crystal-field levels and a fit to calorimetry data from the literature were significantly larger in $\beta$-cerium than in $\gamma$-cerium below room temperature, but the difference was found to be negligible at the experimental phase-transition temperature. A contribution to the specific heat from Kondo spin fluctuations was consistent with the quasielastic magnetic scattering, but the difference between phases was negligible. To be consistent with the latent heat of the $\beta-\gamma$ transition, the increase in vibrational entropy at the phase transition may be accompanied by a decrease in electronic entropy not associated with the crystal-field splitting or spin fluctuations. At least three sources of entropy need to be considered for the $\beta$ - $\gamma$ transition in cerium.
\end{abstract}

DOI: 10.1103/PhysRevB.65.144111

PACS number(s): 64.30.+t, 63.20.-e, 78.70.Nx

\section{INTRODUCTION}

The importance of vibrational entropy to solid-state phase transitions has become well established over the past decade. Considerable experimental ${ }^{1-6}$ and theoretical ${ }^{7-11}$ work has gone into investigating the vibrational entropy of phase transitions in metallic alloys. In alloys, the vibrational entropy is often compared with a significant configurational contribution. For elements, however, entropy can only be vibrational, electronic, and magnetic.

A few recent experimental studies have shown that electronic entropy can also make a significant contribution to high-temperature phase transitions in $f$-electron metals. Inelastic neutron-scattering measurements showed an increase of vibrational entropy during the cerium fcc $(\gamma)$ to bcc $(\delta)$ transition at $1000 \mathrm{~K}$ (Refs. 4 and 6) that was so large that a thermodynamically significant electronic entropy decrease was required to explain the latent heat. Similar measurements on uranium also showed significant electronic contributions-only for uranium the electronic entropy increased in the higher-temperature phases. ${ }^{12}$ For cerium it was suggested that the increase was due mainly to an increase in the electronic density of states at the Fermi level. ${ }^{4}$ Calculations of the electronic density of states of fcc and bodycentered-tetragonal cerium ${ }^{13,14}$ give some support to this suggestion. For uranium, however, the large effects of temperature on the electronic structure ${ }^{12}$ suggest that a more sophisticated approach is needed to explain the electronic entropy. In this work we consider the vibrational and electronic contributions to $\beta$ - and $\gamma$-cerium.

The degenerate electronic states of isolated atoms are split into various crystal-field levels in a crystal. If this crystalfield splitting of electronic levels is of order $k_{B} T$, there is a contribution to the specific heat associated with the partial occupancy of the electronic states. This is seen as the
Schottky anomaly in the specific heat. Crystal-field excitations observed in neutron magnetic scattering can be used to identify the crystal-field energies. Magnetic scattering can be effectively isolated from phonon scattering because it dominates at low angles whereas phonon scattering dominates at high angles. A phase transition can change the local symmetry and the strength of the crystal-field splitting and hence change the entropy. In the present work we compare the $4 f$-electron level splitting of $\beta$ - and $\gamma$-cerium to determine the change in crystal-field entropy. We also consider the contribution from spin fluctuations of the $4 f$-electrons seen as a broadening of the measured crystal-field energy levels. To identify any remaining entropy contribution, we compare the vibrational and crystal-field splitting entropies with the latent heat measured at the $\beta-\gamma$ transition temperature. We deduce that there is a third contribution to the $\beta-\gamma$ transition, probably electronic in origin.

\section{EXPERIMENT}

Two different cold-rolled and annealed plates (approximately $100 \mathrm{~g}$ each) of $99.9+\%$ pure cerium in the $\gamma$ phase were measured at $300 \mathrm{~K}$. For $\beta$-cerium measurements at 150 and $300 \mathrm{~K}$, one of the $\gamma$-cerium plates was transformed to more than $95 \% \beta$-cerium using a thermal cycling technique similar to that described by Koskimaki, Gschneidner, and Jrand Panousis. ${ }^{15}$ The procedure involved cycling from room temperature to $77 \mathrm{~K} 20$ times, annealing at $345 \mathrm{~K}$ for 6 days, and cycling another 20 times.

Neutron-scattering measurements were performed on the LRMECS spectrometer at the Intense Pulsed Neutron Source (IPNS) of the Argonne National Laboratory. The samples were mounted in a closed-cycle helium displex refrigerator. Inelastic measurements were made with incident neutron energies of $E_{i}=45$ and $25 \mathrm{meV}$. The raw data were corrected for self-shielding, sample environment background, detector 


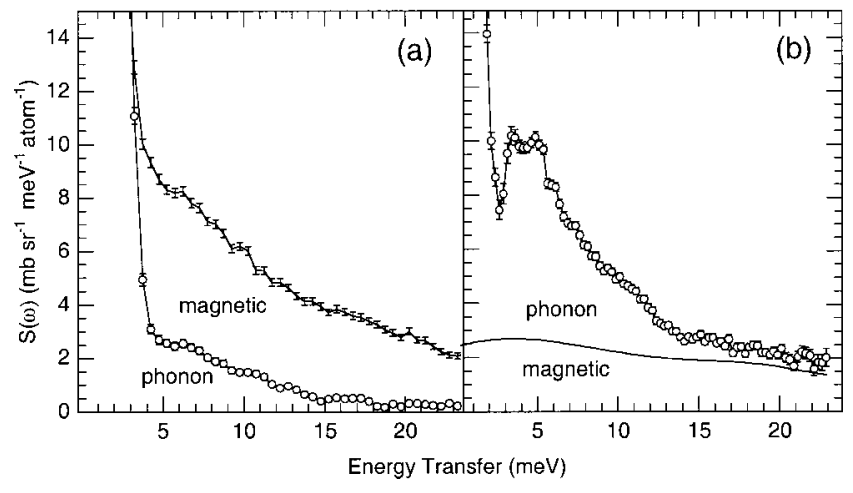

FIG. 1. Phonon and magnetic contributions to $S(\omega)$ for $\beta$-Ce at $300 \mathrm{~K}$; (a) $E_{i}=45 \mathrm{meV}$ and summed over the low angle range $1.95^{\circ}-51.6^{\circ}$, (b) $E_{i}=25 \mathrm{meV}$ and summed over the high angular range $55.3^{\circ}-118.5^{\circ}$. The phonon contribution above the phonon cutoff (about $14 \mathrm{meV}$ ) is from multiphonon scattering.

efficiency, and the $k_{i} / k_{f}$ phase-space factor. The data were normalized in absolute units of millibarns/(steradian $\mathrm{Ce}$ atom) by comparison to a vanadium standard measured under identical spectrometer conditions, giving the scattering function $S(\theta, \omega)$, where $\theta$ is the scattering angle and $\hbar \omega$ the energy transfer.

\section{DATA ANALYSIS}

The analysis of the magnetic scattering is described in detail elsewhere. ${ }^{16}$ The procedure involves summing cerium data over the low angle range from $1.95^{\circ}-51.6^{\circ}$ to increase statistics and minimize the contribution from phonon scattering, which increases with $\theta$. Figure 1(a) shows the magnetic and phonon contributions for the low angle sum at $300 \mathrm{~K}$ and $E_{i}=45 \mathrm{meV}$. The higher-resolution data obtained with $E_{i}$ $=25 \mathrm{meV}$ did not reveal any additional information on the magnetic scattering at $300 \mathrm{~K}$ because the lifetime broadening was much greater than the instrument resolution. The magnetic peak positions were fit using the peak positions inferred from low-temperature measurements and accounting for thermal broadening. ${ }^{16}$ The phonon contribution was approximated using a measured La spectra and accounting for the relative cross sections.

The phonon scattering was studied by summing over the high angle range $55.3^{\circ}-118.5^{\circ}$, where the phonon scattering was largest; and looking at the $300-\mathrm{K}$ data where the magnetic excitations were weak and broadened. Using the magnetic form factor, the fits to the magnetic scattering data were extrapolated from the low angle data to the high angle range and subtracted from the phonon scattering. The size of this magnetic correction is shown in Fig. 1(b).

The incoherent multiphonon scattering was determined iteratively to all orders using a procedure similar to that described by McQueeney. ${ }^{17}$ The procedure involved using a trial phonon density of states (DOS) to calculate the meansquare atomic displacement $\left\langle u^{2}\right\rangle$ and time-dependent selfcorrelation function $G(t)$, where

$$
G(t)=\int_{-\infty}^{\infty} d \omega \frac{g(\omega)}{\omega} n(\omega) \exp (-i \omega t),
$$

$g(\omega)$ is the phonon density of states, and $n(\omega)$ is the thermal occupancy factor. This was used to calculate the total incoherent dynamic structure factor summed over the detector angle $(2 \theta)$ range,

$$
\begin{aligned}
\bar{S}_{\text {calc }}^{\text {inc }}(\omega)= & \sum_{\theta} \frac{1}{2 \pi \hbar} \exp \left[-Q^{2}(\theta, \omega)\left\langle u^{2}\right\rangle\right] \\
& \times \int_{-\infty}^{\infty} d t \exp (-i \omega t) \exp \left(\frac{\hbar^{2} Q^{2}(\theta, \omega) G(t)}{2 m}\right) \\
& \times \exp \left[-\frac{t^{2}}{2}\left(\frac{\Delta E(\omega)}{2 \hbar}\right)^{2}\right],
\end{aligned}
$$

where

$$
Q(\theta, \omega)=\sqrt{\frac{2 M}{\hbar^{2}}\left(2 E_{i}-\hbar \omega-2 E_{i}\left[1-\frac{\hbar \omega}{E_{i}} \cos (2 \theta)\right]^{1 / 2}\right)},
$$

$m$ is the nuclear mass, and $M$ is the neutron mass. The expression in square brackets in Eq. (2) includes a Gaussian instrument energy resolution of variable width, $\Delta E(\omega)$, and minimizes cutoff errors in the numerical Fourier transform. The momentum transfer $Q(\theta, \omega)$ is determined from the experimentally measured scattering angle $\theta$ and the energy transfer $\hbar \omega$.

By expanding the exponential in Eq. (2) containing $G(t)$, the incoherent single phonon and elastic scattering were determined. These were subtracted from the total scattering to give the multiphonon-angle-averaged dynamic structure factor

$$
\bar{S}_{m, \text { calc }}^{\text {inc }}=\bar{S}_{\text {calc }}^{\text {inc }}-\bar{S}_{0, \text { calc }}^{\text {inc }}-\bar{S}_{1, \text { calc }}^{\text {inc }},
$$

where the subscripts $m, 0$, and 1 refer to the multiphonon, elastic, and one-phonon contributions, respectively. Although the previous result was calculated for incoherent scattering, the angle-averaged result is also a good approximation for the multiphonon coherent scattering since the interference terms in the coherent cross section cancel each other to a large extent. ${ }^{18}$ The coherent elastic scattering is just a delta function convoluted with the instrument energy resolution and thus was easily fit and subtracted.

The measured total dynamical structure factor minus the elastic peak was averaged over the detector angles $2 \theta$ and scaled to match $\bar{S}_{\text {calc }}^{\text {inc }}(\omega)-\bar{S}_{0, \text { calc }}^{\text {inc }}(\omega)$. The multiphonon part, Eq. (4), was then subtracted to give an estimate of the onephonon scattering plus a small nearly constant background from multiple scattering, which was removed. The remaining intensity was used to determine a new phonon DOS that was in turn used to recalculate the multiphonon contribution. The procedure was repeated until the phonon DOS converged to within statistical errors. Convergence required three iterations. The final phonon densities of states are shown in Fig. 2 . The $\gamma$-cerium (fcc) and $\beta$-cerium [double hcp (dhcp)] phonon densities of states were essentially identical to the corresponding fcc and dhcp phonon densities of states measured for lanthanum. ${ }^{19}$ For both $\mathrm{Ce}$ and $\mathrm{La}$ there is a shift in intensity to lower energies in the transition from dhcp to fcc near the cutoff energy $(\sim 12 \mathrm{meV})$. 


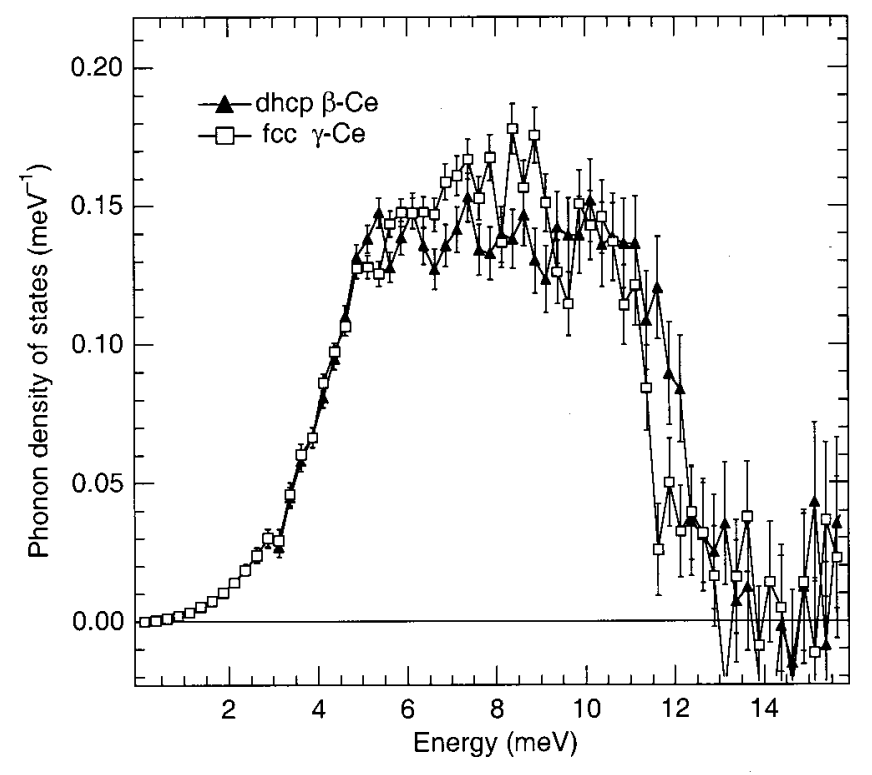

FIG. 2. Phonon DOS curves for $\beta$ - and $\gamma$-cerium at $300 \mathrm{~K}$.

\section{DISCUSSION}

The measured phonon DOS (Fig. 2) was used to calculate the phonon part of the specific heat. The constant volume (harmonic) part of the phonon specific heat was calculated using

$$
C_{V, \mathrm{vib}}(T)=3 k_{B} \int_{0}^{\infty} g(\omega)\left(\frac{\hbar \omega}{k_{B} T}\right)^{2} \frac{\exp \left[\frac{\hbar \omega}{k_{B} T}\right]}{\left.\exp \left[\frac{\hbar \omega}{k_{B} T}\right]-1\right)^{2}} d \omega .
$$

The size of the anharmonic contribution from volume expansion, $C_{p}-C_{V}=9 B \nu \alpha^{2} T$, was estimated using the specific volume $\nu$, bulk modulus $B(0.20 \mathrm{mb}),{ }^{20}$ and the thermal expansion coefficient $\alpha\left(8.1 \times 10^{-6} \mathrm{~K}^{-1}\right)$ (Ref. 4) of $\gamma$-cerium. This contribution was less than the error on the specific heat $(0.1 \mathrm{~J} / \mathrm{mol} \mathrm{K})$ over the temperature range shown and was thus neglected. It is assumed that the anharmonic contributions to $\beta$ - and $\gamma$-cerium are similar in magnitude since they have similar densities and are both close-packed structures differing only in stacking sequence.

The $\gamma$-cerium (fcc) magnetic spectra have a crystal-field excitation at $17 \mathrm{meV} .{ }^{16}$ For a $4 f$ electron with fcc symmetry this corresponds to a transition from a doublet $\left(\Gamma_{7}\right)$ to a quartet $\left(\Gamma_{8}\right){ }^{21}$ The $\beta$-cerium (dhcp) data did not show all the crystal-field peaks because some were too weak and broadened to separate from the quasielastic scattering. The details of this problem are discussed in a separate report on the magnetic scattering in this data set. ${ }^{16}$ The basic problem seems to be a fairly strong hybridization of the $f$ states with the conduction band. This makes accurate predictions of thermodynamic quantities from simple crystal-field models difficult without supporting measurements. Fortunately, calorimetry measurements were made on $\beta$-cerium at low temperatures by Koskimaki and Gschneidner, Jr., ${ }^{22}$ Tsang

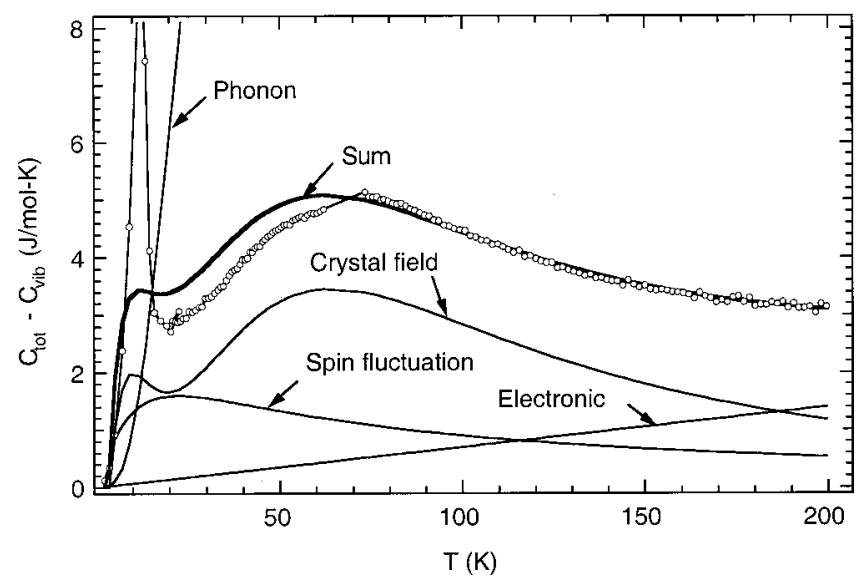

FIG. 3. Phonon-subtracted heat capacity of $\beta$-cerium (circles). Phonon contribution was calculated from the $\beta$-cerium phonon DOS shown in Fig. 2. The crystal-field (Schottky) contribution was calculated from the level scheme determined using the inelastic neutron-scattering spectra (Ref. 16). The spin-fluctuation (Kondo) contribution was calculated with the Coqblin-Schrieffer model (Ref. 28). The thick curve shows the sum of the crystal-field, spinfluctuation, and electronic contributions. The linear electronic contribution was adjusted such that the sum matched the specific heat at high temperatures. Peak at around $10 \mathrm{~K}$ is due to an antiferromagnetic transition. The heat capacity was borrowed from measurements of Koskimaki and Gschneidner, Jr. (Ref. 22), Tsang et al. (Ref. 23), and Gschneidner, Jr. and Pecharsky (Ref. 24).

et al., ${ }^{23}$ and Gschneidner, Jr. and Pecharsky. ${ }^{24}$ After subtracting the phonon part using Eq. (5) we fit the remaining heat capacity by assuming three contributions from the electronic degrees of freedom: (i) crystal field, (ii) spin fluctuations, and (iii) the usual electronic excitations at the Fermi energy. The phonon-subtracted specific heat is shown in Fig. 3.

The $\beta$-cerium dhcp structure has an equal number of sites with distinct cubic and hexagonal site symmetries. The cubic site has the same doublet $\left(\Gamma_{7}\right)$ to quartet $\left(\Gamma_{8}\right)$ 17-meV crystal-field excitation as the fcc structure. ${ }^{16}$ As discussed by McQueeney et al., ${ }^{16}$ it is possible to predict the hexagonal level scheme from the measured cubic levels by following the assumptions of the superposition model. ${ }^{25}$ Briefly, the relevant crystal-field scaling parameters only depend on the polar coordinates of the ligands, which are identical on both the cubic and hexagonal sites. Based on the cubic splitting then the crystal-field level scheme on the hexagonal sites is $\left| \pm \frac{1}{2}\right\rangle$ at $0,\left| \pm \frac{5}{2}\right\rangle$ at 1.9 , and $\left| \pm \frac{3}{2}\right\rangle$ at $9 \mathrm{meV}$. ${ }^{16}$ Thus, if we neglect the effects of lifetime broadening, the crystal-field (CF) specific heat can be determined using

$$
C_{\mathrm{CF}}(T)=\sum_{n}\left[\frac{E_{n}^{2}}{Z(T) k_{B} T^{2}}-\frac{\partial Z(T)}{\partial T} \frac{E_{n}}{Z(T)^{2}}\right] \exp \left(\frac{-E_{n}}{k_{B} T}\right),
$$

where

$$
Z(T)=\sum_{n} \exp \left(\frac{-E_{n}}{k_{B} T}\right)
$$


and $n$ is summed over all levels (half on cubic sites and half on hexagonal sites). The crystal-field specific heat calculated from this scheme is shown in Fig. 3. It should be noted that the lifetime broadening of the crystal-field levels is significant. ${ }^{16}$ A justification for using the simple model of sharp levels is that the specific heat is an integral quantity, so the details of the broadening are smoothed out to a large extent. We do, however, include the broadening in the ground state separately below.

The interaction of the localized $4 f$ electrons with the conduction electrons spreads the energy of the ground-state doublet $\left(\left| \pm \frac{1}{2}\right\rangle\right.$ on the hexagonal sites and $\Gamma_{7}$ on the cubic sites). This can be seen as quasielastic spin fluctuations in the neutron inelastic magnetic spectra. ${ }^{16}$ The spread of these states contributes an additional term to the electronic specific heat. The simplest way to treat this problem is with the Kondo impurity model as is often done with heavy fermion systems. ${ }^{26}$ The problem is in fact very similar, but with a much higher Kondo temperature $\left(T_{K} \sim 40 \mathrm{~K}\right)^{25}$ and a much weaker enhancement of the electronic specific heat at low temperatures. Further support for this approach is that a resistivity anomaly in $\beta$-cerium at around $50 \mathrm{~K}$ has been interpreted successfully in terms of a quenched Kondo scattering mechanism. ${ }^{27}$ Rajan calculated an exact expression for the specific heat using the Coqblin-Schrieffer model. ${ }^{28}$ For the doublet ground states the specific heat from spin fluctuations $(\mathrm{SF})$ is given $\mathrm{by}^{28}$

$$
C_{\mathrm{SF}}(T)=k_{B} \int_{-\infty}^{\infty} \frac{g_{\mathrm{SF}}(\varepsilon)\left(\varepsilon / 2 k_{B} T\right)^{2}}{\cosh ^{2}\left(\varepsilon / 2 k_{B} T\right)} d \varepsilon,
$$

where $g_{\mathrm{SF}}(\varepsilon)$ is the spin-fluctuation density of states that modifies a standard result for a two-level system. We approximate the spin-fluctuation density of states as a Lorentzian with a half width determined from the neutron quasielastic width extrapolated to zero temperature, $\sim 4 \mathrm{meV}^{16}$ The calculated specific heat for this contribution is labeled "spin fluctuation" in Fig. 3. We did not attempt to fit the specific heat at the lowest temperatures because of the antiferromagnetic ordering transition at $\sim 10 \mathrm{~K}$.

If we assume temperatures to be well below the Fermi temperature $T_{f}$, and that the energy derivatives of the electronic DOS can be neglected, the electronic specific heat in the free-electron model can be expressed in terms of the electronic DOS at the Fermi level, $C_{\mathrm{el}}(T)=g\left(\varepsilon_{f}\right)\left(\pi^{2} /\right.$ 3) $k_{B}^{2} T$. Therefore, with these approximations just one adjustable parameter, the electronic specific-heat constant $\gamma$ $=g\left(\varepsilon_{f}\right)\left(\pi^{2} / 3\right) k_{B}^{2}$, was required to fit the remaining electronic contribution to the specific-heat data. The fit, shown in Fig. 3, gives $\gamma=(7.0 \pm 0.1) \mathrm{mJ} / \mathrm{mol} \mathrm{K}^{2}$. With this it is confirmed that temperatures are well below the Fermi temperature since $T_{f}=\left(\pi^{2} / 2\right)\left(k_{B} / \gamma\right)=6060 \mathrm{~K}$. Since the narrow $4 f$ bands result in the largest derivative, our second assumption depends mainly on the location of the $4 f$ bands with respect to the Fermi level. According to Baer and Busch ${ }^{29}$ the $4 f$ bands lie $\sim 900 \mathrm{meV}$ below the Fermi energy in $\gamma$-cerium and thus should not affect the derivatives. More recent results suggest it may be in the $1-2-\mathrm{eV}$ range. ${ }^{30}$ In any case, if the next term in the Sommerfeld expansion was large, an

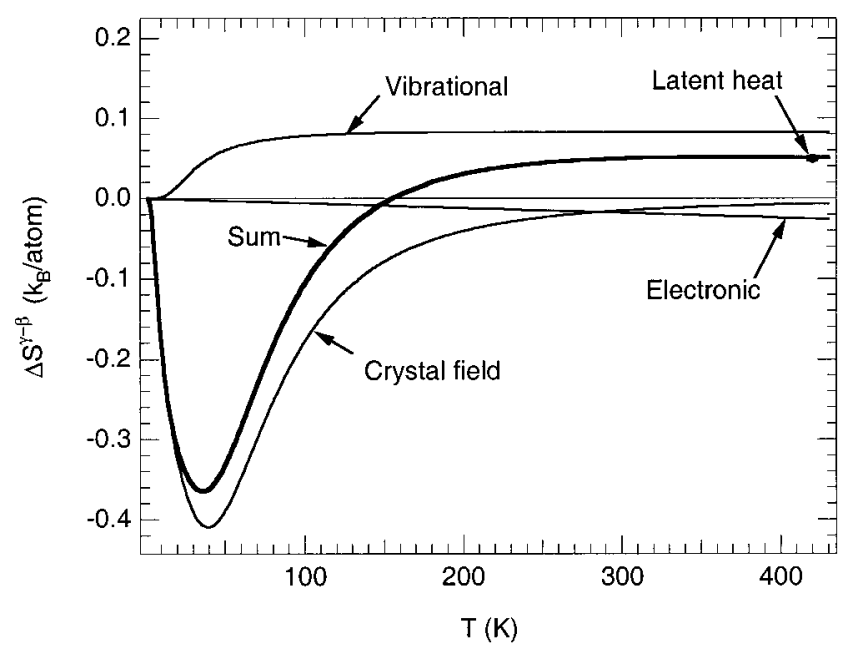

FIG. 4. Crystal-field, vibrational, and electronic contributions to the entropy difference between $\gamma$ - and $\beta$-cerium. Thick curve shows the sum of the three components. The electronic component was adjusted so that the sum equaled the value obtained from the latent heat measurement of Gschneidner, Jr. et al. (Ref. 31).

additional $T^{3}$ term would need to be added to the electronic specific heat. The data did not warrant such a correction.

Despite the simplicity of the models used, the sum of the various electronic components fit the data surprisingly well above the antiferromagnetic transition, Fig. 3. It should be pointed out, however, that many assumptions were not quite correct. In fact, it seems unlikely that the crystal-field picture is even correct in a strict sense since the hybridization with conduction electrons is so strong. ${ }^{16}$ A more accurate model would include the hybridization of each crystal-field state with the conduction electrons. On the other hand, since the specific heat is adequately reproduced the total entropy associated with the electrons must be correct even if the separation into parts is not as clean as the simple models suggest.

The calculated contributions to the specific heat were used to calculate the entropy difference between $\gamma$ - and $\beta$-cerium as a function of temperature using

$$
\Delta S_{i}^{\gamma-\beta}(T)=\int_{0}^{T} \frac{\Delta C_{i}^{\gamma-\beta}}{T} d T
$$

where $i$ indicates the entropy contribution $(i=\mathrm{el}$, vib, CF, and SF). Although the spin-fluctuation part was significant, it made no measurable contribution to the entropy difference because the quasielastic scattering of $\gamma$-cerium is nearly identical to that of $\beta$-cerium. ${ }^{16}$ Crystal-field and vibrational entropy differences are compared in Fig. 4. At the experimental transition temperature $(420 \mathrm{~K})$, the crystal-field contribution is negligible compared to the vibrational contribution. The latent heat measured at $\sim 420 \mathrm{~K}$ implies an entropy change of only $0.05 k_{B}$ /atom, ${ }^{31}$ which is smaller than the change in vibrational entropy. Thus, by setting the sum of the entropy differences equal to the latent heat, Fig. 4 implies an electronic entropy difference of $\Delta S_{\mathrm{el}}^{\gamma-\beta}=-(0.04$ $\pm 0.05) k_{B}$ /atom. This difference is similar to what is found using the electronic specific-heat constants that Koskimaki 
and Gschneidner, $\mathrm{Jr}^{22}$ used to fit $\gamma$ - and $\beta$-cerium calorimetry data $\left(-0.096 k_{B} /\right.$ atom). However, Koskimaki and Gschneidner, $\mathrm{Jr}^{22}$ noted uncertainty in the electronic specific-heat constant for $\beta$-cerium due to the lowtemperature antiferromagnetic transition (Fig. 3). Perhaps more significant was the fact that the spin-fluctuation contribution was not included. At the time of their publication (1974), to our best knowledge, heavy fermions were not known and the calculations of Rajan ${ }^{28}$ did not exist. The neglect of spin fluctuations probably explains why they found such an unusually large value at low temperatures $\left(\sim 46 \mathrm{~mJ} / \mathrm{mol} \mathrm{K}^{2}\right)$ but could not reconcile it with the hightemperature trend. Based on the impurity model with $T_{K}$ $\sim 40 \mathrm{~K}$, the low-temperature limit of the specific heat is of order $C(T \rightarrow 0) / T=\gamma=1.29 \pi k_{B} / 6 T_{K} \sim 100 \mathrm{~mJ} / \mathrm{mol} \mathrm{K}^{2} .{ }^{28}$ It is therefore not surprising that they found a significantly enhanced electronic specific-heat constant at low temperatures.

Because of this uncertainty in the electronic specific heat, the electronic specific-heat constant of $\beta$-cerium (dhcp) was assumed to be equal to that of dhcp lanthanum. ${ }^{22}$ The present results make no such assumption and thus imply independent estimates of the electronic specific-heat constants: (7.0 $\pm 0.1) \mathrm{mJ} / \mathrm{mol} \mathrm{K}^{2}$ for $\beta$-cerium from the fit in Fig. 3 and $(6.2 \pm 0.8) \mathrm{mJ} / \mathrm{mol} \mathrm{K}^{2}$ for $\gamma$-cerium after accounting for the latent heat and other entropy terms. Compared with the values used by Koskimaki and Gschneidner, Jr., ${ }^{21} 9.4$ $\mathrm{mJ} / \mathrm{mol} \mathrm{K}^{2}$ for $\beta$-cerium (which is actually just the lanthanum value) and $7.5 \mathrm{~mJ} / \mathrm{mol} \mathrm{K}^{2}$ for $\gamma$-cerium, our results are slightly smaller, but their difference is similar.

The crystal-field and electronic entropies tend to stabilize the $\gamma$ phase with respect to the $\beta$ phase at low temperatures. Although the crystal-field entropy difference is negligible at the measured transition temperature $(420 \mathrm{~K})$, the crystal-field entropy difference becomes important at the lower (true) transformation temperature, $283 \mathrm{~K}$, determined in a 20 -year study by Gschneidner, Jr. et al. ${ }^{31}$ Of course in a 20 -year study it is not possible to measure the latent heat and, thus, determine the total entropy change. However, inspection of Fig. 4 shows that the entropy difference is essentially the same in magnitude as at $420 \mathrm{~K}$ but that the crystal-field entropy assumes a more significant role with respect to the electronic entropy (from continuous excitation of electrons across the Fermi energy).

\section{SUMMARY}

Many competing sources of vibrational and electronic entropy need to be included in the equation of state of cerium. The contributions of the vibrational and electronic degrees of freedom to the entropy of the $\beta$ and $\gamma$ phases of cerium were determined. Their different temperature dependencies change their relative importance. At the experimentally observed transition temperature $(\sim 420 \mathrm{~K})$, a vibrational entropy difference of $\Delta S_{\mathrm{vib}}^{\gamma-\beta}=(0.09 \pm 0.05) k_{B} /$ atom is dominant, followed by the electronic contribution $\Delta S_{\mathrm{el}}^{\gamma-\beta}=-(0.04$ $\pm 0.05) k_{B} /$ atom, and then a negligible crystal-field contribution. The crystal-field entropy difference dominates at low temperatures, and is comparable to the electronic contribution at the true transition temperature $283 \mathrm{~K}$. A contribution from spin fluctuations was determined, but made no difference between the two phases.

\section{ACKNOWLEDGMENTS}

The authors would like to thank Professor K. A. Gschneidner for lending us the calorimetry data. This work was supported by the U.S. Department of Energy under Contract No. W-7405-ENG-36 with the University of California and Grant No. DE-FG03-96ER45572 with the California Institute of Technology. This work has benefited from the use of the IPNS at Argonne National Laboratory. This facility is funded by the U.S. Department of Energy, under Contract No. W-31-109-ENG-38.
${ }^{1}$ L. Anthony, L. J. Nagel, J. K. Okamoto, and B. Fultz, Phys. Rev. Lett. 73, 3034 (1994).

${ }^{2}$ B. Fultz, L. Anthony, L. J. Nagel, R. M. Nicklow, and S. Spooner, Phys. Rev. B 52, 3315 (1995).

${ }^{3}$ L. J. Nagel, B. Fultz, and J. L. Robertson, Philos. Mag. B 50, 7291 (1997).

${ }^{4}$ J. L. Robertson, H. N. Frase, P. D. Bogdanoff, M. E. Manley, B. Fultz, and R. J. McQueeney, Philos. Mag. Lett. 79, 297 (1999).

${ }^{5}$ M. E. Manley, B. Fultz, and L. J. Nagel, Philos. Mag. B 80, 1167 (2000)

${ }^{6}$ M. E. Manley, R. J. McQueeney, J. L. Robertson, B. Fultz, and D. A. Neumann, Philos. Mag. Lett. 80, 591 (2000).

${ }^{7}$ A. F. Guillermet and G. Grimvall, J. Phys. Chem. Solids 53, 105 (1992).

${ }^{8}$ S. J. Clark and G. J. Ackland, Phys. Rev. B 48, 10899 (1993).

${ }^{9}$ G. D. Garbulsky and G. Ceder, Phys. Rev. B 53, 8993 (1996).

${ }^{10}$ A. Van de Walle, G. Ceder, and U. V. Waghmare, Phys. Rev. Lett. 80, 4911 (1998).
${ }^{11}$ C. Wolverton, V. Ozolins, and A. Zunger, Phys. Rev. B 57, 4332 (1998).

${ }^{12}$ M. E. Manley, B. Fultz, R. J. McQueeney, C. M. Brown, W. L. Hults, J. L. Smith, D. J. Thoma, R. Osborn, and J. L. Robertson, Phys. Rev. Lett. 86, 3076 (2001).

${ }^{13}$ O. Eriksson, J. M. Wills, and A. J. Boring, Phys. Rev. B 46, 12981 (1992).

${ }^{14}$ P. Ravindran, L. Nordstrom, R. Ahuja, J. M. Wills, B. Johansson, and O. Eriksson, Phys. Rev. B 57, 2091 (1998).

${ }^{15}$ D. C. Koskimaki, K. A. Gschneidner, and N. T. Jrand Panousis, J. Cryst. Growth 22, 225 (1974).

${ }^{16}$ R. J. McQueeney, M. E. Manley, B. Fultz, G. Kwei, R. Osborn, and P. Bogdanoff, Philos. Mag. B 81, 675 (2001).

${ }^{17}$ R. J. McQueeney, Ph.D. thesis, University of Pennsylvania, 1996.

${ }^{18}$ G. L. Squires, Introduction to the Theory of Thermal Neutron Scattering (Dover, NY, 1978).

${ }^{19}$ N. Nücker, in Proceedings of the International Conference on Lattice Dynamics, 1977, edited by M. Balkanski (Paris, Flammarion, 1978), p. 244. 
${ }^{20}$ F. F. Voronov, L. F. Vereshchagin, and V. A. Goncharorva, Dokl. Akad. Nauk SSSR 135, 1104 (1960) [Sov. Phys. Dokl. 135, 1280 (1960)].

${ }^{21}$ K. R. Lea, M. J. M. Leask, and W. P. Wolf, J. Phys. Chem. Solids 23, 1381 (1962).

${ }^{22}$ D. C. Koskimaki and K. A. Gschneidner, Jr., Phys. Rev. B 10, 2055 (1974).

${ }^{23}$ T. W. E. Tsang, K. A. Gschneidner, Jr., D. C. Koskimaki, and J. O. Moorman, Phys. Rev. B 14, 4447 (1976).

${ }^{24}$ K. A. Gschneidner, Jr. and V. K. Pecharsky, J. Phase Equilib. 20, 612 (1999).

${ }^{25}$ D. J. Newman and B. Ng, Rep. Prog. Phys. 52, 699 (1989).
${ }^{26}$ A. Yatskar, W. P. Beyermann, R. Movsovich, and P. C. Canfield, Phys. Rev. Lett. 77, 3637 (1996).

${ }^{27}$ S. J. Liu, P. Burgardt, K. A. Gschneidner, and S. Legvold, J. Phys. F: Met. Phys. 6, L55 (1976).

${ }^{28}$ V. T. Rajan, Phys. Rev. Lett. 51, 308 (1983).

${ }^{29}$ Y. Baer and G. Busch, J. Electron Spectrosc. Relat. Phenom. 5, 611 (1974).

${ }^{30}$ J. Röhler, Handbook on the Physics and Chemistry of Rare Earths, edited by K. A. Gschneidner, Jr., L. Eyring, and S. Hufner (Elsevier, New York, 1987), Vol. 10, p. 453.

${ }^{31}$ K. A. Gschneidner, Jr., V. K. Pecharsky, Jaephil Cho, and S. W. Martin, Scr. Mater. 34, 1717 (1996). 